\title{
Clinicopathological spectrum of fungal infections in a Tertiary Care Centre
}

\author{
M. Sridevi ${ }^{1, *}$, C. Vimala ${ }^{2}$, S. Chitra ${ }^{3}$ \\ ${ }^{\mathbf{1}}$ Associate Professor, ${ }^{2}$ Resident, ${ }^{3}$ Head \& Professor, Dept. of Pathology, Saveetha University, Chennai, Tamil
} Nadu, India

*Corresponding Author:

Email: dr.sridevi81@gmail.com

\begin{abstract}
Introduction: Fungal lesions are common in immunocompromised patients like Diabetic Mellitus, patients on anticancer drug and radiotherapy, HIV and transplant recipients. Although tissue culture being the gold standard for diagnosing fungal infections, histopathological examination gives better idea about tissue invasion.

Materials and Methods: This study was done over 47 cases for a period of four years. This study was done to analyze the clinicopathological spectrum of fungal infections, morphology and their distribution according to age, sex and site of involvement in histopathological specimens in our hospital setup.

Results: In this study, the most common fungal organism was found to be Phaeohypomycosis and most of these cases of phaeohypomycosis presented as ganglion and dermoid cyst .Most common site was foot.

Conclusion: In this study we attempted to determine the etiological diagnosis of fungal infections in histopathological specimens received in our department.
\end{abstract}

Keywords: Fungal lesions, Dermoid cyst, Ganglion, Gomori methenamine silver stain, Immunocompromised patients, Phaeohypomycosis.

Received: $06^{\text {th }}$ March, 2017

Accepted: $28^{\text {th }}$ September, 2017

\section{Introduction}

Fungal infections have become more common nowadays which causes a wide range of diseases in humans. They range from superficial infections involving the stratum corneum of the skin to systemic infection involving the brain, heart, lungs, liver, spleen, and kidneys. Invasive fungal lesions are more common in patients like transplant receipients, cancer patients on chemotherapy and immunosuppresive treatment, HIV patients, premature infants, old aged patients and those undergoing major surgeries. These patients are at high risk for acquiring invasive fungal infections. ${ }^{1,2}$

Fungal infections based on the site of infection can be superficial limited to stratum corneum, cutaneous involving the skin appendages, subcutaneous or deep involving the abdominal viscera, lungs, bones and the central nervous system. They can also be classified based on the route of acquisitions and type of virulence. Deep mycoses are either caused by primary pathogenic or opportunistic fungal pathogens. The primary pathogenic fungi can infect a normal host and their of entry is through the respiratory system, whereas, opportunistic pathogens infect immunocompromised patients and invade through the respiratory tract, alimentary tract, or intravascular devices. ${ }^{1,3}$
Tissue culture is the gold standard for diagnosing fungal organisms. But there are few instances where the morphological characteristics of fungi are specific like in Pneumocystis and Rhinospoidiosis, they can only be diagnosed by histopathological examination and not by culture. For providing a definitive diagnosis of an invasive fungal infection histopathology continues to be a costeffective and rapid diagnostic technique. Similarly, for evaluating tissue invasion and host reaction to the organism's histopathology is mandatory. ${ }^{4,5}$ Hence histologic evaluation of tissue sections is a quick and easy way for identifying fungal organisms, and also a strong adjunct to microbiologic culture in diagnosing fungal infections. ${ }^{1,6}$

In superficial mycosis usaully there won't be any inflammation. Whereas in cutaneous infection inflammation is elicited in the epidermis and around the hair and nail. And in subcutaneous infection the inflammation develops first in the subcutaneous tissue and later may extend into the epidermis. To determine the specific agent present in the histopathologic specimen various other techniques may be used like immunohistochemistry, in situ hybridization, and PCR. ${ }^{2,7}$

The aim of this study was to analyze the clinicopathological spectrum of fungal infections, morphology and their distribution 
according to age, sex and site of involvement in histopathological specimens in our hospital setup.

\section{Materials and Methods}

This study was done in the department of pathology for a period of four years from January 2012 to March 2016. A total of 47 cases were included in this study. In every case sex, site of lesion, and clinical diagnosis were noted down. Small biopsy specimens were all embedded and in large specimens maximum number of bits were taken. All the sections were stained with hematoxylin and eosin $(\mathrm{H} \& \mathrm{E})$ and wherever required special stains for fungus like Gomori methenamine silver (GMS) stain and Periodic acid Schiff stain. All cases were histologically studied for the morphological identification of the fungus, presence of necrosis, granuloma, calcification and inflammation.

In all cases of the histologic evaluation of granulomatous lesions we used special stains for identifying fungus and Ziel neilsons stain for excluding the presence of acid fast bacilli. Usually in the tissue section, fungi occur either as hyphae, or budding yeast, or spherules, or a combination of these forms. Hematoxylin \& Eosin stain enables us to evaluate the host response, as well as to detect other microorganisms. Whenever the fungi are sparsely distributed,or poorly stained, there are chance for missing them in H\&E stained sections. In case of certain fungal lesions like Histoplasmosis and Blastomycosis the morphologic features may be misleading and may not be evident as they may show retraction artifact in the cytoplasm, or may show variation in the sizes making morphologic evaluation difficult. Sometimes some of the fungi can have pseudohyphae, or the therapy may alter the fungal morphology in tissue section. Hence Special stains for fungi are essential for histopathologic evaluation of unexplained inflammatory lesions. Gomori Methenamine Silver (GMS) and Periodic acidSchiff (PAS) are the two most common special stains used for the identification of fungus. Gomori's methenamine silver (GMS), and periodic acid-Schiff (PAS), are called as "broad spectrum" fungal stains. Even in case where fungi are degenerated and non viable, GMS gives better contrast hence it is highly preferred for screening. ${ }^{4,8,9}$

\section{Result}

During the study period, of 15682 total histopathological specimens, we got 47 cases of fungal infections accounting for $0.2 \%$ of total histopathological specimens. Out of these 47 cases, 17 cases were female and 30 cases were male with male predominance. The age group ranged from 10 to 80 years with a mean age of 45 yrs. Phaeohypomycosis constitutes a significant proportion of all fungal infections seen in 17 cases of our study followed by 7 cases of aspergillosis and others were 5 cases of candidiasis, 4 cases of Mucormycosis, 3 cases each of Maduramycosis, and Rhinosporidiosis, 2 cases of Dermatophytosis and one case of Sporotrichosis. In five cases, specific etiology was not possible and was labeled as positive for fungal elements in four cases and one case as sub cutaneous mycosis.

The most common site of infection was foot seen in 13 cases, followed by hand and nasal cavity. In 14 cases there was necrosis, in 24 cases granuloma formation, 18 cases showed marked inflammation and in one case calcification was seen. Table 1,2 and 3 summarizes the clinical presentations, histopathological diagnosis and host response to mycosis. Fig. 1 to 5 summarizes the morphologies of various fungus.

Table 1: Spectrum of clinical presentation

\begin{tabular}{|l|c|c|}
\hline & Frequency & Percentage \\
\hline CNS & 2 & 4.3 \\
\hline Nasal Cavity & 7 & 13 \\
\hline Maxilla & 1 & 2.3 \\
\hline Paranasal Sinus & 3 & 6.8 \\
\hline Wrist & 3 & 6.5 \\
\hline Elbow & 2 & 4.3 \\
\hline Forearm & 2 & 4.3 \\
\hline Hand & 7 & 13 \\
\hline Foot & 13 & 27.6 \\
\hline Leg & 4 & 8.6 \\
\hline Thigh & 1 & 2.1 \\
\hline Ankle & 1 & 2.1 \\
\hline
\end{tabular}




\begin{tabular}{|l|c|c|}
\hline Skin Plaque & 1 & 2.1 \\
\hline Total & 47 & 100 \\
\hline
\end{tabular}

Table 2: Histopathological diagnosis

\begin{tabular}{|l|c|c|}
\hline & Frequency & Percentage \\
\hline Phaeohypomycosis & 17 & 38.2 \\
\hline Aspergillosis & 7 & 15.2 \\
\hline Candida & 3 & 6. \\
\hline Dermatophytosis & 2 & 4.3 \\
\hline Maduramycosis & 3 & 6.5 \\
\hline Mucormycosis & 2 & 4.3 \\
\hline Actinomycosis & 3 & 6.5 \\
\hline Positive For Fungal Elements & 4 & 8.6 \\
\hline Rhinosporidiosis & 3 & 6.5 \\
\hline Sporotrichosis & 1 & 2.1 \\
\hline Subcutaneous Mycosis & 1 & 2.1 \\
\hline Total & 47 & 100 \\
\hline
\end{tabular}

Table 3: Host response to Mycosis

\begin{tabular}{|l|c|c|}
\hline & Frequency & Percentage \\
\hline Necrosis & 14 & 29.7 \\
\hline Granuloma & 24 & 51 \\
\hline Marked inflammation & 18 & 38.29 \\
\hline Calcification & 1 & 2.1 \\
\hline
\end{tabular}

Table 4: Clinical diagnosis of Phaeohypomycosis

\begin{tabular}{|l|c|}
\hline Clinical Diagnosis & No. of Cases \\
\hline Ganglion & 6 \\
\hline Dermoid Cyst & 5 \\
\hline Abscess & 1 \\
\hline Dermatofibrosarcoma & 1 \\
\hline Sebaceous Cyst & 2 \\
\hline Cyst & 1 \\
\hline Swelling & 1 \\
\hline Total & 17 \\
\hline
\end{tabular}

Table 5: Study comparison to the literature

\begin{tabular}{|l|l|l|l|}
\hline & Present Study & $\begin{array}{l}\text { Muniyappa } \\
\text { Usha et al }^{\mathbf{3}}\end{array}$ & $\begin{array}{l}\text { Sidhaling reddy } \\
\text { et al }^{\mathbf{2}}\end{array}$ \\
\hline Sex & M>F & M>F & M>F \\
\hline $\begin{array}{l}\text { Age } \\
\text { incidence }\end{array}$ & $10-80 \mathrm{yrs}$ & $10-70 \mathrm{yrs}$ & $10-60 \mathrm{yrs}$ \\
\hline $\begin{array}{l}\text { Common } \\
\text { organism }\end{array}$ & $\begin{array}{l}\text { Phaeohypomy- } \\
\text { cosis }\end{array}$ & Mucormycosis & Rhinosporidiosis \\
\hline $\begin{array}{l}\text { Common } \\
\text { site }\end{array}$ & Foot & $\begin{array}{l}\text { Maxillary } \\
\text { sinus }\end{array}$ & Nasal cavity \\
\hline
\end{tabular}

Phaeohyphomycosis is caused by dematiaceous fungi with cytoplasmic melanin like brown to black pigments fungi. Most of the cases of phaeohypomycosis presented as ganglion and dermoid cyst and they also had areas of necrosis with one case had calcification. One case of phaeohypomycosis was clinically diagnosed as dermatofibrosarcoma.

\section{Discussion}

Histopathology examination helps in identification of infectious fungal organisms and remains the reliable method of identifying certain pathogens. Microbiological examination cannot differentiate contamination of fungi from colonizing pathogenic fungus. Some fungi cannot be cultured. Guarner and Brandt and 
Sundaram et al have shown the difficulties in differentiating colonization and contamination of fungi. ${ }^{2,10}$

In our study out of 47 cases of fungal infections, accounting for $0.2 \%$ of total histopathological specimens, Phaeohypomycosis constitute a significant proportion of all fungal infections.

Phaeohyphomycosis is a collective term for a heterogeneous group of fungal infections caused by dematiaceous fungi and the commonly isolated ones are Exophiala jeanselmei, E. spinifera, and Wangiella dermatitidis. They lead to slowly progressive infections of the superficial, cutaneous, subcutaneous and deep visceral tissues and are identified by the presence of pigmented yeast- like and hyphae forms but no sclerotic cells or muriform cells or copper pennies as seen in Chromoblastomycosis (Picture A). These infections are life-threatening, and occur in various immunocompromised patients. ${ }^{1,3,5,11-14}$

In phaeohyphomycosis, the cyst wall histopathologically consists of fibro- collagenous tissue and granulomatous inflammation with many giant cells. Fungal elements in the form of yeast-like structures and septated hyphae were found throughout the lesions. They presented mostly as ganglion cyst and sebaceous cyst and histologically showed tissue reaction in the form of necrosis, inflammation, granuloma and calcification. Histopathology method helps in etiologic diagnosis; it can detect fungal infections in clinically unsuspected cases. Like in our study, six cases of ganglion cyst and five cases of dermoid cyst and one case of clinically suspected dermatofibrosarcoma was found to be Phaeohypomycosis. Table- 4 summarizes the clinical diagnosis in 17 cases of pheohypomycosis.

In our study the next common fungi was Aspergillosis seen in seven cases. Two cases of aspergillosis presented as space occupying lesion of the brain. Aspergillosis were identified by thin acute-angled dichotomous branching hyphae which are septated (Picture B). Usually the hyphae will be deeply basophilic, whereas degenerated or necrotic hyphae will appear hyaline or eosinophilic. Aspergillosis fumigatus is the most common species and A. niger can cause disease in immunosuppressed hosts. Aspergillosis includes three entities: allergic bronchopulmonary aspergillosis (ABPA), chronic pulmonary aspergillosis /aspergilloma, and invasive or systemic aspergillosis., ,, $15^{-15}$

In ABPA, the lesions may show granulomatous inflammation, vasculitis, interstitial fibrosis, and microabscesses with scanty hyphae which could represent an invasive aspergillosis. Aspergilloma is a mass of fungal hyphae with a necrotic background. They form fungal ball or fruiting body which is formed in the preexisting pulmonary cavity due to tuberculosis. These cavities are usually surrounded by fibrosis. Sometimes may show granuloma with Splendore - Hoeppli phenomenon. Fungus balls are sometimes produced by some other fungi, like Coccidioides immitis and Pseudallescheria boydii. Typical hyphae of the Aspergillus species are uniform, narrow and regularly septate. Similarly their branching is also regular, dichotomous and acute angled. Invasive aspergillosis involves the lungs and paranasal sinuses and disseminate to the brain, liver,kidneys, heart, and bones.

We had five cases of candidiasis (Picture C), all were middle aged females with one case presented in nasal cavity. Candida albicans is the most common species causing candidiasis. It usually colonises the vagina and oropharynx. It can cause either superficial or deep invasive infection in case of microbial imbalance due to use of antibiotics, hormones and in immunocompromised patients like diabetic, cancer patient or HIV patients. Microscopically, Candida appear as yeasts intermingled with pseudohyphae with periodic constrictions. C. glabrata does not form pseudohyphae. Invasion of tissues and blood vessel indicates invasive candidiasis and that helps in differentiating from colonization. ${ }^{1,2,4,5,15}$

In our study, Mucormycosis was seen in three cases (Picture D). Out of which one case was clinically diagnosed as maxillary carcinoma. They are diagnosed by irregular broad hyphae branching at right angle. Hyphae can be sometimes septate or nonseptate, as it appears in most of the time. The hyphae are often folded, twisted, wrinkled, or collapsed as they have little structural stability. Sometimes ovoid forms of chlamidoconidia are seen in the tissues. Mucormycosis may clinically present as rhinoorbito-cerebral, pulmonary, cutaneous, gastrointestinal or disseminated infection. Mucormycosis is common in immunocompromised patients and occurs rarely in immunocompetent hosts following trauma. ${ }^{2,4,5,15}$

We had three cases of Maduramycosis (Picture E) Out of which one case had an unusual presentation in the wrist. Usually mycetoma can be classified as eumycotic and actinomycotic mycetoma. Maduramycosis is characterized by the presence of draining sinus tracts with visible pigmented brown to black grains composed of 
microcolonies of fungi which causes the infection. ${ }^{1,2,15}$

Rhinosporidiosis was seen in three cases.R. seeberi is the most common species causing this rhinosporidiosis, characterized by polyploid tumors in the nose, nasopharynx, and ocular region. They are identified by the round sporangium with endospores in various stages. Rarely they may disseminate to other mucous membranes, skin and viscera. ${ }^{2,4,15}$

We reported two cases of Dermatophytosis. Dermatophytes are identified in tissue sections by their spores(arthroconidia) and branched, septate hyphae which invade the stratum corneum, hair follicle, hair shafts and nails. They includes three entities : Epidermophyton, Trichophyton, and Microsporum.

Epidermophyton usually involves skin and nails with no involvement of hair shafts and follicles.

Microsporum involves hair and skin with no nail involvement.

Trichophyton usually infects hair, skin, and nails.

As the dermatophytes have hyaline hyphae they are best demonstrated with GMS and PAS stains. In the stratum corneum they are seen in between upper normal and lower abnormal appearing stratum corneum. The hair invasion may be in the following pattern: ectothrix , endothrix or endoectothrix. They can lead to severe neutrophilic inflammation of hair follicles and shafts in the form of kerion or severe mononuclear inflammation in the form of Majocchi's granuloma. ${ }^{1,2,4}$

We had one case of sporotrichosis, which is caused by sporothrix schenckii. Microscopically they appear as round, or oval, or cigar-shaped yeasts that may show tube-like budding. S. schenckii yeasts are not easily identified with H\&E stains, and hence GMS and PAS stains should be used to identify them. In cases of sporotrichosis, asteroid bodies may be seen, which are star shaped, eosinophilic material surrounding the yeasts and Sporothrix has been demonstrated in these asteroid bodies using immunohistochemistry. ${ }^{1,2,5}$

We compared our study with the two other studies and found that Phaehypomycosis was the most common organism in our study, whereas in Muniyappa Usha et al, ${ }^{4}$ Mucormycosis and in Sidhaling reddy et $\mathrm{al}^{7}$ Rhinosporidiosis were found to be common as shown in Table 5 . Similarly in our study foot was the commonest site of fungal infection, whereas in Muniyappa Usha et al, maxillary sinus and in Sidhaling reddy et al, nasal cavity was found to be the commonest site.

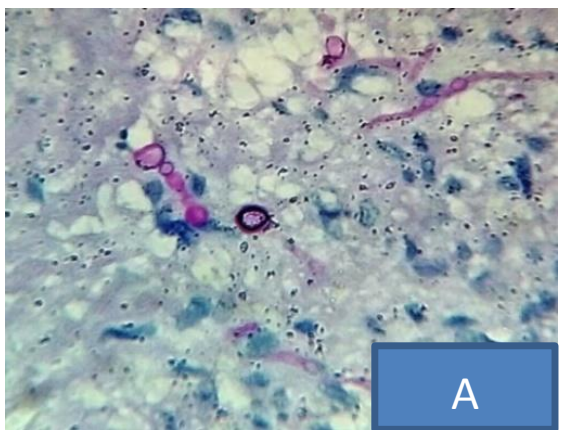

A: Pheohypomycosis, pigmented hyphae and yeast forms 400x (H\&E)

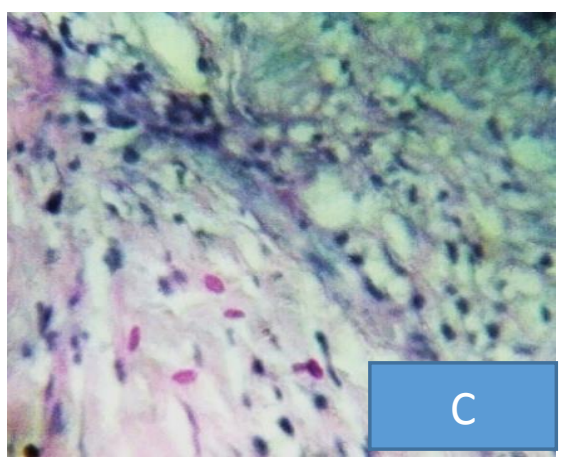

C: Candidiasis, Yeast forms 400x (PAS)

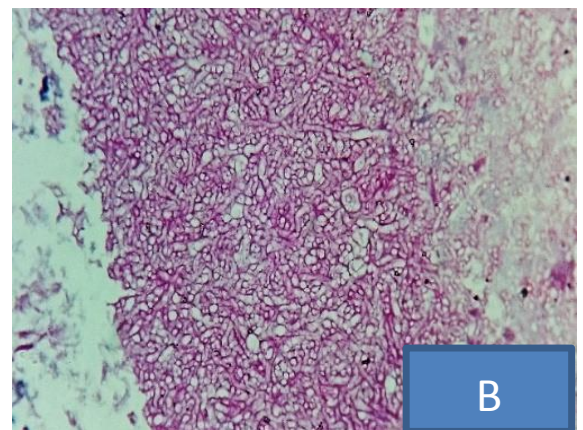

B: Aspergillosis, thin acute angled septate hyphae forming fruiting body 400X (PAS)

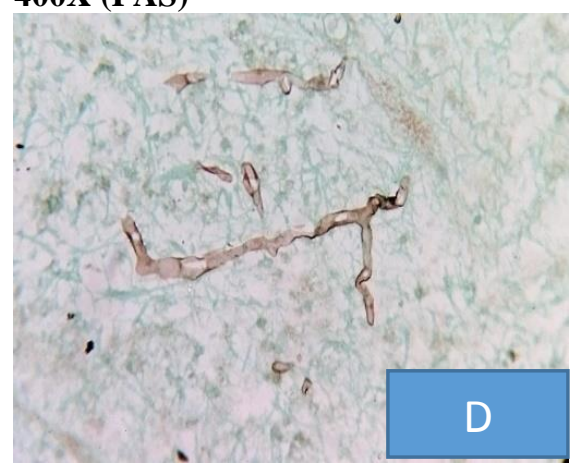

D: Mucormycosis, non septate right angled broad branching hyphae 400x (GMS) 


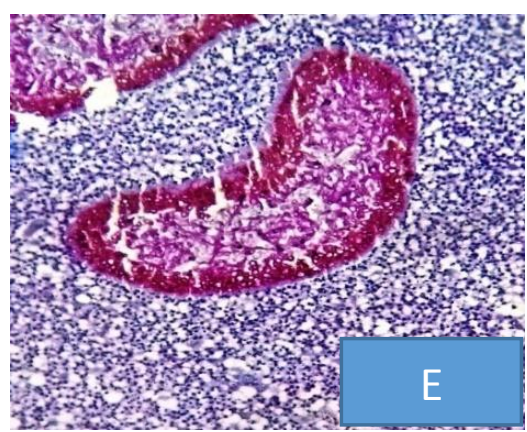

\section{E: Neutrophilic abscess with center granule of Maduramycosis bordered with Splendore-Hoeppli material 400X (PAS)}

\section{Conclusion}

In this study we attempted to determine the etiological diagnosis of fungal infections in histopathological specimens received in our department. The histopathologic examination can thus offer reliable means to identify pathogenic fungus and the host reaction.

\section{References}

1. Walsh TJ, Dixon DM. "Spectrum of Mycoses". In: Baron S, editor. Medical Microbiology. 4th edition. Galveston (TX): University of Texas Medical Branch at Galveston; 1996. Chapter 75.

2. Jeannette Guarner and Mary E. Brandt, "Histopathologic Diagnosis of Fungal Infections in the $21 \mathrm{st}$

3. Century" Clinical Microbiology Reviews (2011) April 24(2),247-80.

4. Kobayashi GS. Disease of Mechanisms of Fungi. In: Baron S, editor. Medical Microbiology. 4th edition. Galveston (TX): University of Texas Medical Branch at Galveston; 1996. Chapter 74.

5. Muniyappa Usha, HB Rakshitha, Jasuja Avnika, Asok Aneesha, Reginald Sharon, "Morphological spectrum of fungal infections: a retrospective study" International Journal of Medical Science and Public Health (2016) 5(8),1673-7.

6. Vicky J. Schnadig, Gail L.Woods, "Histopathology of fungal infections"Clinical mycology, 2nd edition. Churchil Livingstone Elsevier,79-108.

7. Sangoi AR, Rogers WM, Longacre TA, Montoya JG, Baron EJ, Banaei N, "Challenges and pitfalls of morphologic identification of fungal infections in histologic and cytologic specimens: a ten-year retrospective review at a single institution"
American Journal of Clinical Pathology (2009) 131(3),364-75.

8. Sidhalingreddy, Deepa Masur, Arun T. Miskin, V.D.Domble, Jayashree Patil, "Histopathological spectrum of fungal infections in a tertiary care centre" International Journal of Biological and Medical Research (2013) 4(1),2889-93.

9. Abida Haque, "Special Stains Use in Fungal Infections" Connection (2010)187-94.

10. Sarah M. Heaton, Amy C. Weintrob, Kevin Downing, Bryan Keenan, Deepak Aggarwal, Faraz Shaikhand. "Histopathological techniques for the diagnosis of combat-related invasive fungal wound

11. infections"BioMedCenter Clinical Pathology (2016)16:11.

12. Sundaram C, Umabala P, Laxmi V, Purohit AK, Prasad VS, Panigrahi M, et al, "Pathology of fungal infections of the central nervous system: 17 years' experience from Southern India" Histopathology (2006):49(4), 396-405.

13. Ana Sofia Castro, Ana Oliveira and Virginia Lopes, "Pulmonary phaeohyphomycosis: a challenge to the clinician" European Respiratory Review (2013):22(128),187-92.

14. A. W. Fothergill,"Identification of Dematiaceous Fungi and Their Role in Human Disease"

15. Clinical Infectious Diseases (1996) 22(8uppl 2):8179-84

16. Barenfanger J, Ramirez F, Tewari R, et al, "Pulmonary phaeohyphomycosis in a patient with hemoptysis" Chest (1989) 95,1158-60.

17. Shantha Ravisankar, R. Vimal Chander, "Cerebral pheohyphomycosis: Report of a rare case with review of literature" Neurology India (2013):61(5),1-2.

18. P. P. Anthony, "A guide to the histological identification of fungi in tissues" Journal of clinical Pathology (1973):26,828-31. 